\title{
Occurrence and Distribution of Cryptococcus Species in Environmental Sources from Lower Assam Belt of India
}

\author{
Nurul Islam ${ }^{1} \mathbb{D}$, Rupjyoti Bharali ${ }^{1}$, Sailen Talukdar $^{1} \mathbb{D}$, Syed Akram Hussain ${ }^{2} \mathbb{D}$, \\ Afzal Hoque Akand ${ }^{3}$ iD and Hridip Kumar Sarma ${ }^{*}$ (D) \\ ${ }^{1}$ Laboratory of Microbial communication and Fungal Biology, Department of Biotechnology, Gauhati University, \\ Guwahati-781014, Assam, India. \\ 2Division of Veterinary Public Health, Faculty of Veterinary Sciences and Animal Husbandry, SKUAST-Kashmir, \\ Shuhama, Srinagar-19006, India. \\ ${ }^{3}$ Division of Veterinary and Animal Husbandry Extension, Faculty of Veterinary Sciences and Animal Husbandry, \\ SKUAST-Kashmir, Shuhama, Srinagar-19006, India.
}

*Correspondence: hridip@gauhati.ac.in; +91 9613861702

(Received: September 15, 2020; accepted: December 08, 2020)

Citation: Islam N, Bharali R, Talukdar S, Hussain SA, Akand AH, Sarma HK. Occurrence and Distribution of Cryptococcus Species in Environmental Sources of Lower Assam Belt of India. J Pure Appl Microbiol. 2020;14(4):2781-2800. doi: 10.22207/JPAM.14.4.54

C The Author(s) 2020. Open Access. This article is distributed under the terms of the Creative Commons Attribution 4.0 International License which permits unrestricted use, sharing, distribution, and reproduction in any medium, provided you give appropriate credit to the original author(s) and the source, provide a link to the Creative Commons license, and indicate if changes were made. 


\begin{abstract}
Cryptococcus is a non-motile, gram positive, non-fermenting Basidiomycetous encapsulated yeast like fungus that causes respiratory, neurological and other systemic diseases in both humans and animals. Present study delineates the possible distribution of Cryptococcus species in pigeon droppings, excreta of other avian species, eucalyptus tree and contaminated soil specimens collected from different geographical co-ordinates of six geographical regions of the lower Brahmaputra Valley of Assam, India. The fungi were isolated through conventional methods of Sabouraud Dextrose Agar (SDA) and Bird Seed Agar (BSA) media and identified through negative staining of capsule as well as performing classical bio-chemical tests. Identity of the isolates was further confirmed through sequence analysis of ITS-1 and ITS-4 region of the 18S rDNA. Two pathogenic species of Cryptococcus were isolated from $67(15.40 \%)$ of the 435 specimens. Of these positive isolates 41 (9.43\%) belonged to Naganishia albida (Cryptococcus albidus) while 26 (5.98\%) represented Papiliotrema laurentii (Cryptococcus laurentii). Both the species were recovered from 58 (18.35\%) dry and 9 (7.56\%) moist specimens. The percentage of prevalence of Naganishia albida in dry and moist specimens were $35(11.07 \%)$ and 6 (5.04\%) respectively. Contrary to this, the percentage of prevalence of Papiliotrema laurentii in dry and moist were $23(7.28 \%)$ and 3 (2.52\%) respectively. The findings indicate that Cryptococcus species have established a better ecological sustenance in dry specimens than moist. The findings of the investigation demonstrated that the prevalence of Cryptococcus albidus in attics, dovecotes / houses of pigeon fanciers, contaminated soil, eucalyptus tree and droppings of other birds were $11(12.36 \%)$ out of 89 , $23(14.11 \%)$ of $163,2(3.23 \%)$ of $62,4(7.84 \%)$ of 51 and only $1(1.43 \%)$ out of 7 specimens respectively. The recovery of Papiliotrema laurentii in the above specimens were $3(3.37 \%), 20(12.27 \%), 1(1.61 \%)$, $1(1.96 \%)$ and $1(1.42 \%)$ respectively. The findings revealed that the prevalence of Naganishia albida is more than that of Papiliotrema laurentii in natural substrates. The notorious pathogenic fungi, Cryptococcus neoformans could not be isolated, indicative of the fact that the region selected for the study is not environmentally favorable for growth and sustenance of the species. Findings of the study clearly demonstrate the ecological and epidemiological significance of the non-neoformans species of the genus cryptococcus that needs further comprehensive studies to access the prevalence of the genus from public health point of view.
\end{abstract}

Keyword: Cryptococcus, pigeon droppings, natural substrates, ecological relationship, lower Brahmaputra valley, prevalence

\section{INTRODUCTION}

Cryptococcus is non-motile, gram positive, non-fermenting Basidiomycota from the order Tremellale under the family of Tremellaceae of capsulated yeast like fungus ${ }^{1}$. Littman (1959) reported the first case of cryptococcosis in man directly attributed to pigeon excreta ${ }^{2}$. Outbreaks of cryptococcosis are reported in human, animals and avian species whilst presence of the causative organism has been reported in reptiles, fruits and vegetables ${ }^{3}$. Of more than 70 species of the genus Cryptococcus, a majority thrive in the environment and only few of them are medically important disease causing pathogenic agents ${ }^{4}$. Amongst all the species, Cryptococcus neoformans is regarded as the major human and animal pathogen, while Cryptococcus albidus and Cryptococcus laurentii are occasionally known to cause moderate to severe diseases especially in immuno-compromised patients ${ }^{5}$.
Cryptococcus species are commonly found in dropping of pigeons that apparently harbors the organism in normal commensal form ${ }^{6}$. In addition, the digestive tracts of parrots and canaries also harbour the fungus ${ }^{7}$. Pigeon droppings, are available in most unlikely places such as roofs and ventilations of abandoned buildings, cornices, leaves and branches of trees that serve as ecological niche for adaptation, dispersion of Cryptococcus replication and transmission. Other environmental sources such as fruits and vegetables retain the fungi as saprophyte that may cause infection in man and animals either by inhalation of spores of the organism or through subcutaneous inoculation 3,8-10.

Excreta of pigeon as saprobic reservoir of Cryptococcus species have been frequently recovered from various countries of the world ${ }^{3,11,12}$. Isolation of Cryptococcus from many tree 
species in America ${ }^{13}$ Brazil ${ }^{14}$, India ${ }^{15}$, Iran ${ }^{16}$ have been reported.

Among the systemic and opportunistic mycoses, cryptococcosis continues to exacerbate severe health risks, especially in high risk groups and immuno-compromised patients. It has been estimated that the global prevalence of cryptococcosis among AIDS patients stands at $2.33 \%$ and $6.8 \%$ both worldwide and within India respectively ${ }^{3}$. However, incidence of cryptococcosis in recent years is alarmingly increasing at a global scale amounting to one million infections and approximately 6,25,000 deaths annually, rendering the disease to be considered as an most important fungal diseases 17.

Cryptococcosis is a highly infectious and enigmatic mycotic disease that affect a variety of animals too ${ }^{18,19}$. The disease occurs in acute, sub-acute or in chronic forms and have global significance ${ }^{20}$. The fungi causes respiratory as well as neurological problems and is often sporadic in nature ${ }^{19,21,22}$. Incidentally, the exact epidemiological data on the incidence and prevalence of the disease is not readily available as the disease is not a notifiable one although several reports of considerable morbidity and mortality in human as well as in animals are well documented 21.

The first report of Cryptococcosis in India was documented in 1952 and subsequently many other investigators reported its occurrence from time to time in various parts of the country ${ }^{23}$. Although numerous studies on the zoonotic importance and epidemiology of Cryptococcus neoformans have been reported, yet there is lack of proper information as literatures are scanty on the non-neoformans species like Cryptococcus albidus, Cryptococcus laurentii or Cryptococcus uniguttulatus ${ }^{7}$. In recent years, a burst of increase in opportunistic infection by these non-neoformans pathogenic yeasts have been observed ${ }^{24}$. Environmental sources including canopy leftovers from some trees are regarded as the main sources of Cryptococcus albidus, Cryptococcus laurentii, and Cryptococcus uniguttulatus that lead to infection in human and animals $16,25,26$.

Epidemiology of non-neoformans Cryptococcus albidus, Cryptococcus laurentii,
Cryptococcus uniguttulatus, Cryptococcus luteolus and some other species of the genus are highly relevant as these species often turn out to be pathogenic and thereby increases the risk of infection. A case of encephalitis in an HIV patient due to Cryptococcus albidus was reported in China ${ }^{27}$ while fungal keratitis due to the same species was reported by Huang et.al, (2015) ${ }^{28}$. Earlier, pulmonary Cryptococcosis due to Cryptococcus laurentii in diabetis and patients suffering from ganglio-neuroblastoma were reported by Averbuch et.al, (2002) ${ }^{29}$ and Shankar et.al, (2006) ${ }^{30}$ respectively. Reports are also available on Cryptococcal meningitis, Cryptococcal myelitis and multiple skin lesions in HIV patients infected with Cryptococcus neoformans var. grubii from Assam having a history of occupational exposure to pigeons and chicken ${ }^{31}$.

Such findings are of greater significance to places like Assam where domestication of pigeons, fowls and various birds of the finch family are common among the inhabitants at a large scale both as household pets and for meat. A proper surveillance of the sites of occurrence of the fungi as well as characterization of its diverse forms for creating a database is a primary need since the practice of rearing pigeons is non-scientific, traditional and sporadic reports of pulmonary diseases (including cryptococcal meningitis) have been recorded from time to time amongst the common mass. There is a remarkable gap of information on this aspect in the entire northeastern part of India, including Assam. This is a preliminary attempt for identifying the hot spots of Cyptococcus occurrence in environmental samples representing the lower Brahmaputra valley of Assam and characterizing the isolates for their proper identification to generate a baseline data. It is envisaged that the study will generate information for future planned research activities on the opportunistic systemic mycotic disease causing fungi.

\section{MATERIALS AND METHOD}

In the present investigation, a total of 435 samples of pigeon droppings and droppings of other avian species, barks and leaf litter of natural stands of eucalyptus trees as well as environmental soil from different sources from five different distinct regions of the lower Brahmaputra 
Valley of Assam were collected. These included the districts of Bongaigaon, Dhubri -(both north bank extending up to West Bengal border and south bank extending upto the border of the state of Meghalaya along the river Brahmaputra), the district of Kokrajhar BTAD region, the district of Goalpara extending up to the border with Meghalaya, the district of greater Kamrup (including both urban and rural extensions along the north bank of the river Brahmaputra) and finally the district of Nalbari of western lower Assam. Samples collected were transported to the laboratory in ice cooled boxes packed in polyethylene bags. However, cloacal samples of pigeon were collected using sterile cotton swab in a transport media (Eurotubor, Rubi Barcelona, Spain). After collection, all samples were either processed on same day or stored at $4^{\circ} \mathrm{C}$ until being processed for isolating Cryptococcus species. In the meantime, basic environmental characteristics of the collection areas were noted for understanding the ecological and epidemiological relevance of the organism collected from different sources (Table.1, Fig.1, Fig.2 and Fig.3). The marked samples were processed as per standard methods mentioned elsewhere with slight modifications 14,32 .

\section{Isolation and Identification}

Processing of samples was done in the Department of Biotechnology, Gauhati University. Two grams $(2 \mathrm{~g})$ of the samples was diluted in 20 $\mathrm{ml}$ of sterile physiological saline and the mixture was stirred for five minutes in a vortex apparatus and allowed to stand for 15-20 minutes for

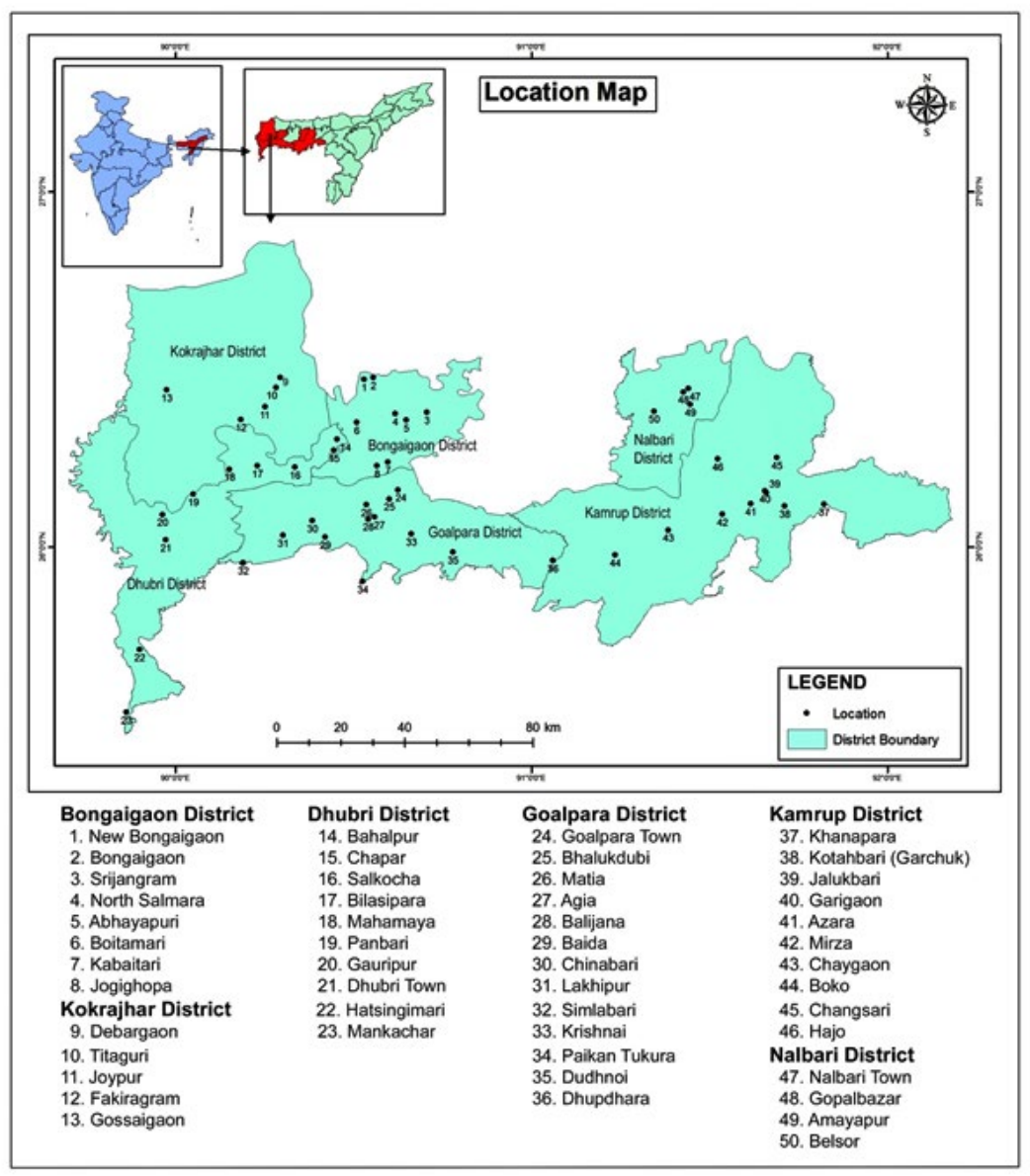

Fig. 1. Representation of sample collection areas developed from GPS coordinates and using GIS software prepared by Aaranyak, Guwahati, Assam 
decantation. After this, $1 \mathrm{ml}$ of the supernatant was inoculated onto $9 \mathrm{ml}$ of sterile physiological saline supplemented with chloramphenicol $(0.05 \mathrm{mg} / \mathrm{ml})$ and then incubated at $37^{\circ} \mathrm{C}$ for an hour. An aliquot of $0.1 \mathrm{ml}$ of each supernatant of the processed samples were aspirated and streaked onto duplicate plates of Sabouraud Dextrose Agar (SDA) and a Bird Seed Agar (BSA) supplemented with chloramphenicol $(0.05 \mathrm{mg} / \mathrm{ml})$. Cultures with SDA media were incubated at $37^{\circ} \mathrm{C}$ while that of Bird Seed Agar (BSA) were incubated at $25^{\circ} \mathrm{C}$ for 15 days and monitored daily from $2^{\text {nd }}$ day onward for observing growth and presence of colony and to evaluate colony morphology. Brown pigmented colonies were identified as Cryptococcus neoformans while colonies showing cream colouration with smooth and mucoid appearance were identified as non-neoformans species coglomerate. Results evaluated on the basis of morphological data were expressed as the average nos. of viable yeast cells per gram of sample (CFU/g). For comparisons, Cryptococcus laurentii MTCC 2898 (procured from CSIR Institute of Microbial technology, Chandigarh - 1600361, India) was used as control. Identified isolates were sub cultured, purified and further subjected to an array of morphological, cultural and bio-chemical tests such as carbohydrates, nitrite reduction, urease production and phenol oxidation ${ }^{11,15,16,20,24}$.

\section{Capsule Identification}

For capsule identification of the probable Cryptococccus spp., a drop of inoculum from newly inoculated isolates grown on SDA was added onto India ink stain on a sterile glass slide with a cover slip and observed under a bright

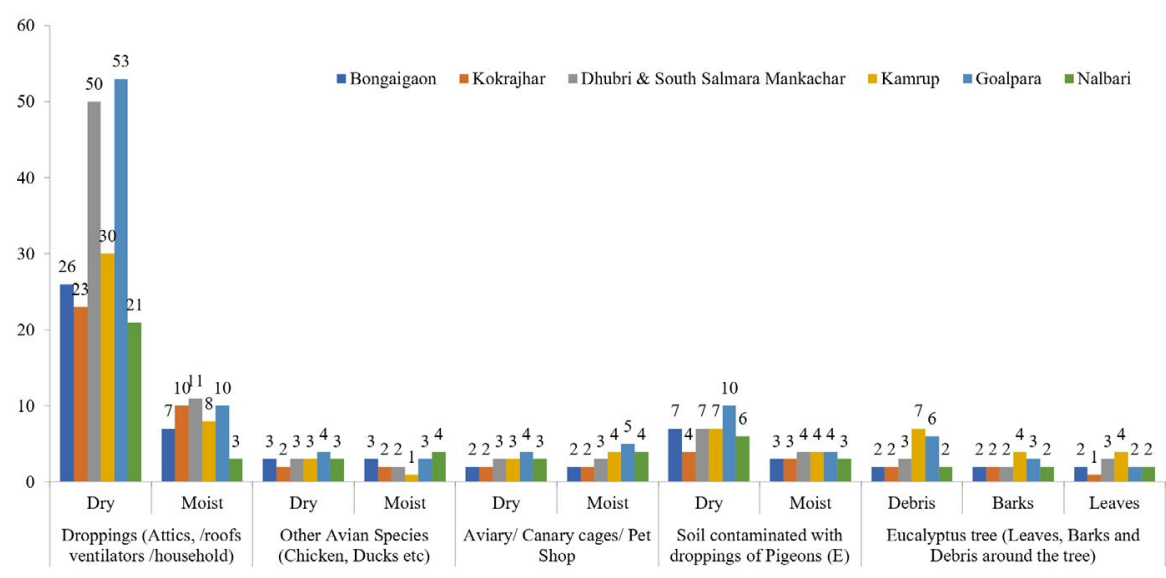

Fig. 2. Frequency distribution of samples as per their sources and types

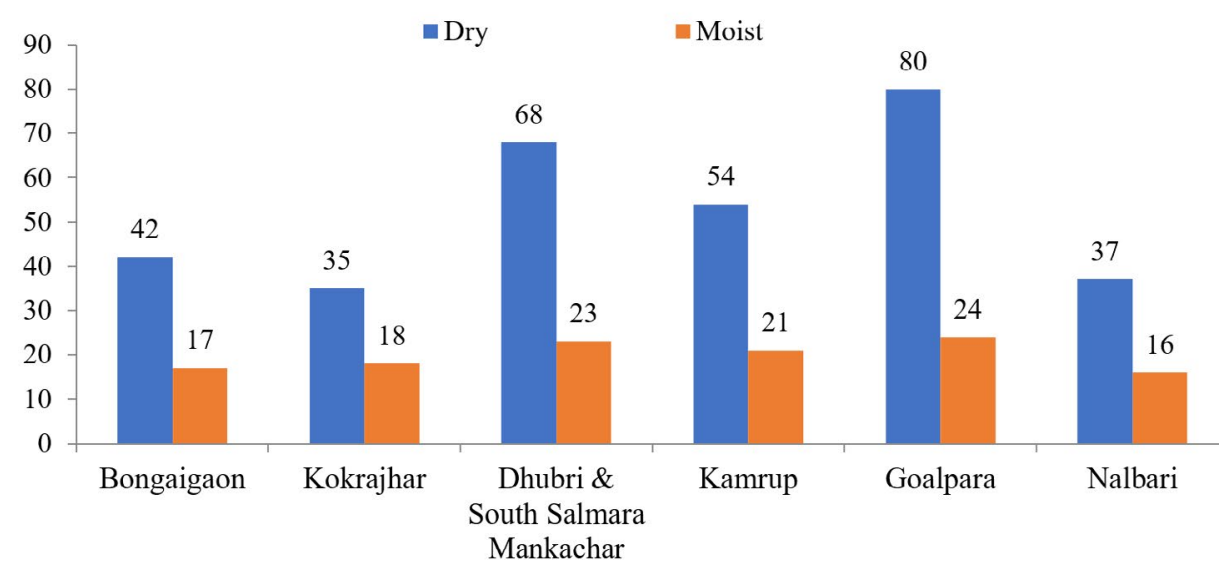

Fig. 3. Distribution of samples collected from different regions and their type 
field microscope (Olympus CX33) at 100 and 400 times magnification (Kwon-Chung and Bennett, 1992) and also phase contrast microscopy (Leica DM750) under 1000 fold magnification. Presence of distinct wide round to oval gelatinous capsule, with or without hyphae was considered as positive observation ${ }^{33-36}$.

\section{Molecular Characterization \\ DNA extraction}

For DNA extraction, isolates from pure cultures were inoculated onto $1.5 \mathrm{ml}$ Eppendorf tubes containing $0.5 \mathrm{ml}$ of Sabouraud Dextrose broth supplemented with chloramphenicol and incubated overnight in an orbital shaker at $150 \mathrm{rpm}$ and $30^{\circ} \mathrm{C}$. After 24 hours, the fungal suspensions with predetermined concentrations were centrifuged at 5,000 rpm for 10 minutes, and the pellet was frozen at $-20^{\circ} \mathrm{C}$ for 1 hour with further incubation at $65^{\circ} \mathrm{C}$ for $1 \mathrm{~h}$ in 0.5 $\mathrm{ml}$ of extraction buffer containing $50 \mathrm{mM}$ Tris$\mathrm{HCl}, 50 \mathrm{mM}$ EDTA, 3\% sodium dodecyl sulfate and 1\% 2-mercaptoethanol (Ferrer et al., 2001). The lysate was finally extracted with phenolchloroform-isoamyl alcohol (v/v) in the ratio $25: 24: 1$. To this, $65 \mu$ of $3 \mathrm{M}$ sodium acetate and $75 \mu \mathrm{l}$ of $1 \mathrm{M} \mathrm{NaCl}$ were added and the resulting volume was incubated at $4^{\circ} \mathrm{C}$ for 30 minutes. DNA was recovered by isopropanol precipitation and washed with $70 \%(\mathrm{v} / \mathrm{v})$ ethanol. Concentration of DNA was measured at $260 \mathrm{~nm}$ in a UV-VIS Spectrophotometer (Shimadzu) and stored at $-20^{\circ} \mathrm{C}$ until further use ${ }^{37-39}$.

\section{PCR amplification}

For characterization of Cryptococcus species, isolated DNA was amplified in a gradient PCR (Eppendorf Nexus Gradient). The primers

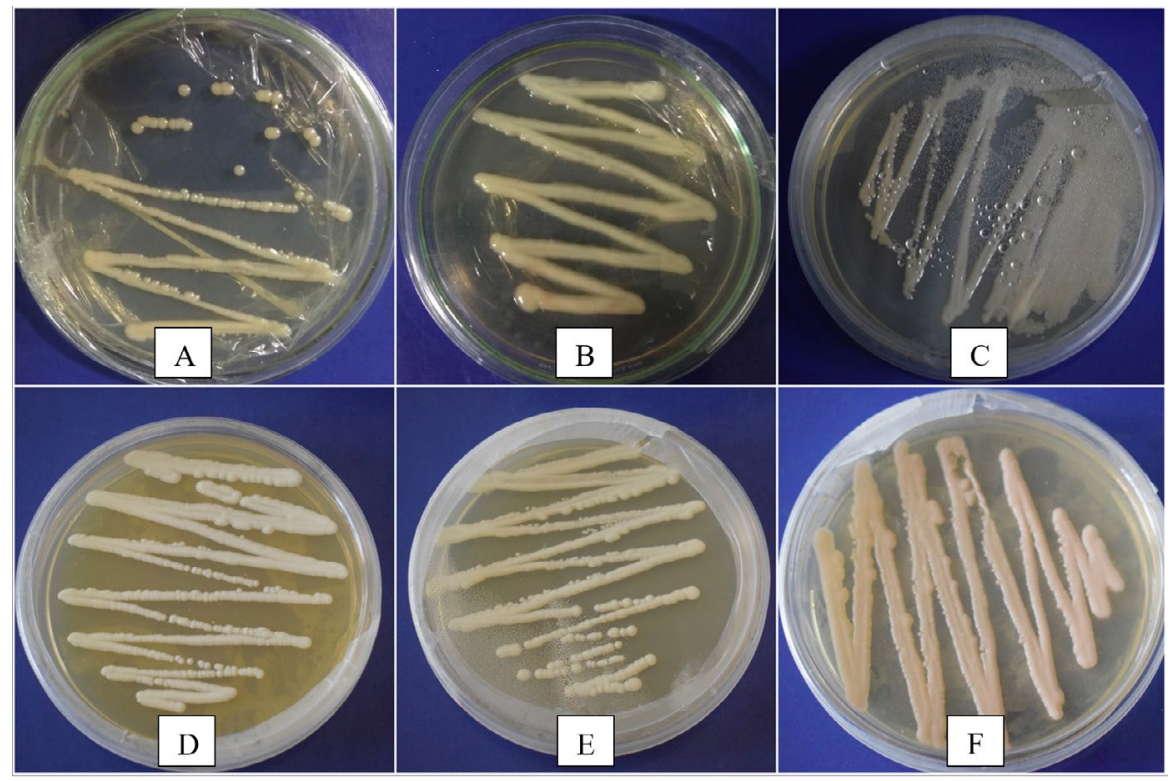

Fig. 4. ((A) Reference strain ) Papiliotrema laurentii (MTCC 2898), (B, C) Papiliotrema laurentii, (D, E, F) Naganishia albida.

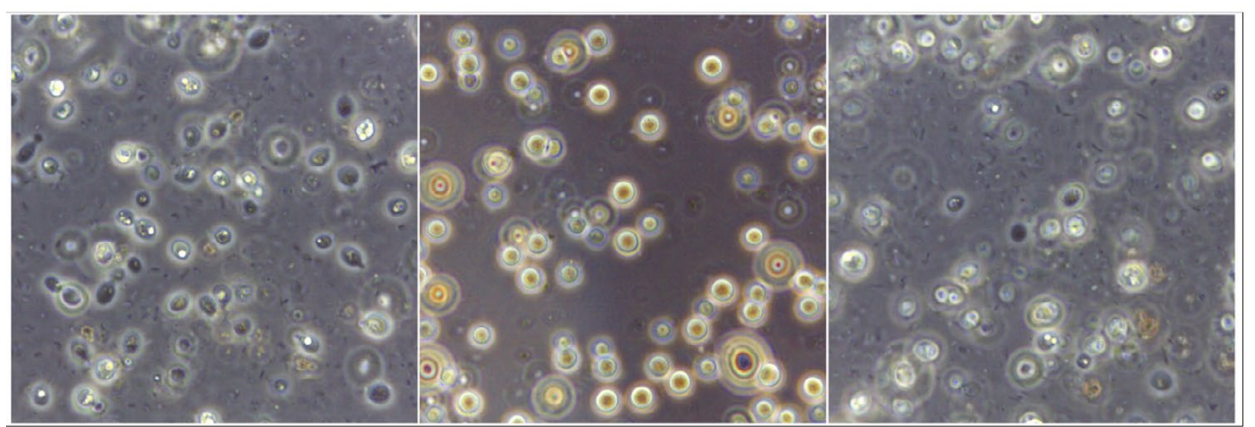

Fig. 5. Phase contrast microscopy of reference sample - Papiliotrema laurentii (MTCC 2898), under 100X magnification 


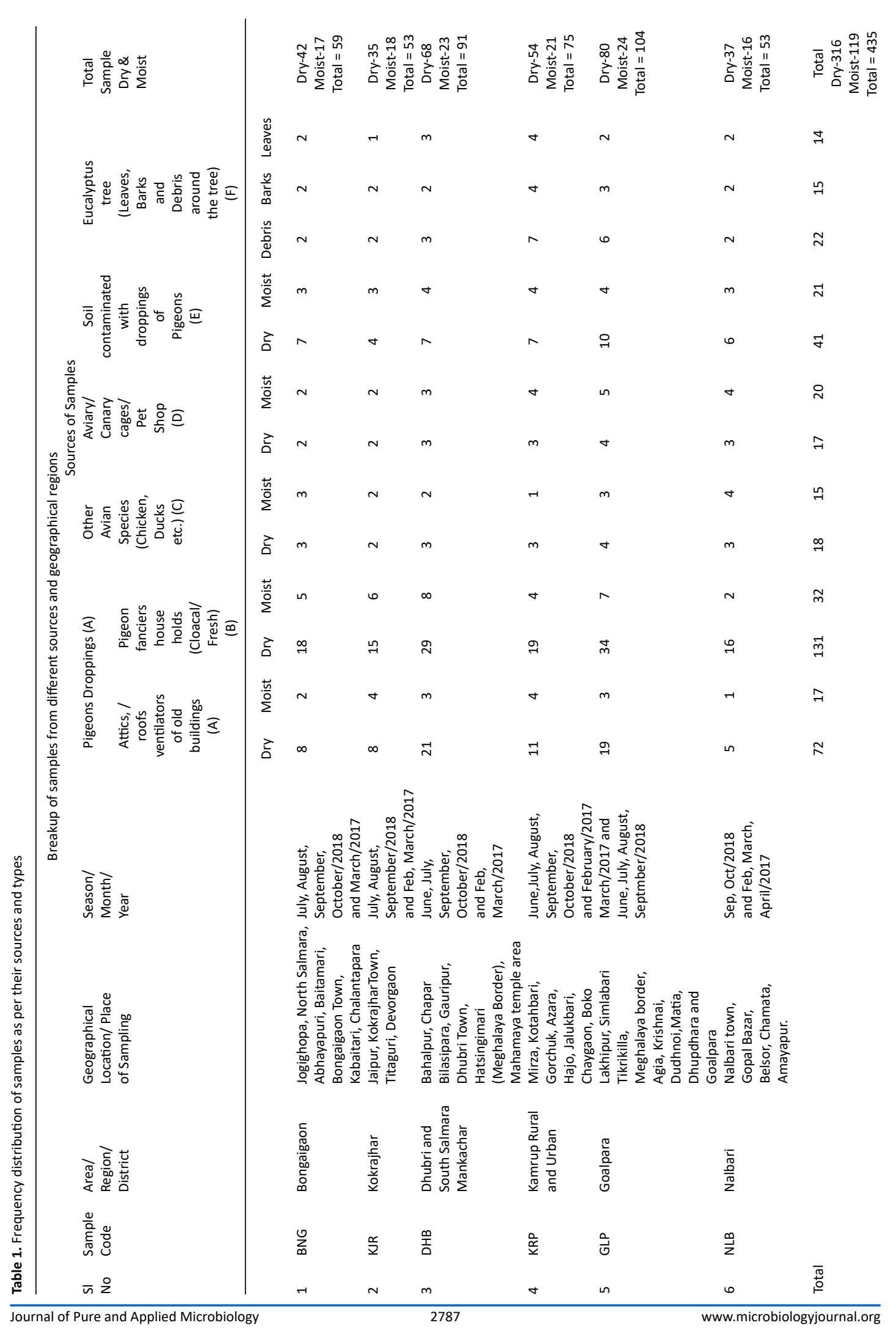


used for amplification included the D1/D2 regions targeting ITS1 and ITS4 with expected fragment length of $600 \mathrm{bp}$. Details of the primers are given () in Table 2. The program for amplification was set for 1 cycle of initial denaturation for 30 minutes at $94^{\circ} \mathrm{C}$ followed by 35 cycles of denaturation for 1 minute at $94^{\circ} \mathrm{C}$, primer annealing for 1 minute at $55^{\circ} \mathrm{C}$, chain extension for 1 minute at $72^{\circ} \mathrm{C}$ and a final extension for 7 minutes at $72^{\circ} \mathrm{C}$ respectively. The PCR products after amplification were resolved in a $1.5 \%$ agarose gel subjected to electrophoresis and was visualized under UV gel documentation system (UVitec Cambridge, Genei). The amplicons were later stored at $-20^{\circ} \mathrm{C}$ for further analysis ${ }^{40-43}$. Sequencing

Sequencing of PCR products were done at Xcelris Labs Limited, India through outsourcing. Chromatogram files obtained were analyzed for nucleotide-BLAST on NCBI portal for identification of the species. Phylogram and dendrogram was prepared using PhyML (Phylogenetic Maximum Likelihood) (http://www.atgc-montpellier.fr/ phyml/).

\section{Statistical analysis}

A very brief descriptive analysis was carried out to interpret the data as they were mostly qualitative attributes. However, positive results obtained from different sources were compared through k-proportion test through Monte Carlo / Marascuilo methods to assess their homogeneity across sources with the help of XLSTAT software ${ }^{44-46}$.

\section{RESULTS}

In the present study, a total of 67 Cryptococcus spp were recovered from 435 samples with a prevalence of $15.40 \%$ (Table 3). Out of 67 positive isolates, $41(9.43 \%)$ were identified as Cryptococcus albidus (Naganishia albida) Table 3, Fig. 4, Plate-D, E, F while 26 (5.98\%) were identified as Cryptococcus laurentii (Papiliotrema

Table 2. Sequence of primers used for PCR amplification

\begin{tabular}{lc}
\hline Primer & Sequence \\
\hline ITS1 & 3'- TCC GTA GGT GAA CCT GCG G -5' \\
ITS4 & 5'- TCC TCC GTC TAT TGA TAT GC -3'
\end{tabular}

laurentii). Details of the identified (Table. 3, Fig. 4, Plate-B, C). Cultural and biochemical characteristics of Naganishia albida and Papiliotrema laurentii were almost similar except in the utilization of potassium nitrate which was negative in case of Papiliotrema laurentii. Cultural characteristics of Cryptococcus laurentii (Fig. 4, Plate B, C) in SDA media were comparable with the reference strain MTCC 2898 (Fig. 4, Plate A). Based on the type of sample (dry and moist), Naganishia albida (Cryptococcus albidus) and Papiliotrema laurentii (Cryptococcus laurentii) could be isolated from 58 (18.35\%) of dry and 9 (7.56\%) of moist specimens collected from different geographical niches / coordinates. (Table 3, Fig. 7).

During the investigation of 57 (22.61\%) Cryptococcus species isolated from pigeon droppings from sites A \& B, 34 (13.49\%) were identified to be Cryptococcus albidus while 23 (9.13\%) represented Cryptococcus laurentii. The highest recovery percentage of Cryptococcus (57) was observed in pigeon droppings and the least (2) was from avian species other than pigeon. In terms of relative isolation of positive cultures of Cryptococcus, 14 (15.73\%) were from attics / ventilations / old and abandoned buildings (Site A), 43 (26.38\%) were from houses of pigeon fanciers / dovecots (Site B), 3(4.84\%) were from contaminated environment / soil (Site C), $5(9.80 \%)$ were from the debris / barks / leaves of eucalyptus trees (Site D) while $2(2.86 \%)$ were from other avian sources like chicken, duck, parrot etc (Site E). The results are presented in Table 3.

Relative and absolute percentage of isolates of Cryptococcus (Naganishia albida and Papiliotrema laurentii) from each collection site was also analyzed. Prevalence rate of isolates from Site A (3.22\%), Site B (9.89\%), Site C (0.69\%), Site $D(1.15 \%)$ and Site $E(0.46 \%)$ was recorded (Table 4). Results interpret that there exist a significant difference at $0.05 \%$ among the variants of different proportions obtained from various samples during the study. Values having the superscript across different rows do not differ significantly.

The overall prevalence of Naganishia albida, across all dry and most environmental samples was $9.43 \%$ (41 of 435 ). However, the prevalence of the species was $35(11.07 \%)$ in dried specimens in comparison to 6 (5.04\%) from moist specimens. Details of prevalence of Naganishia 


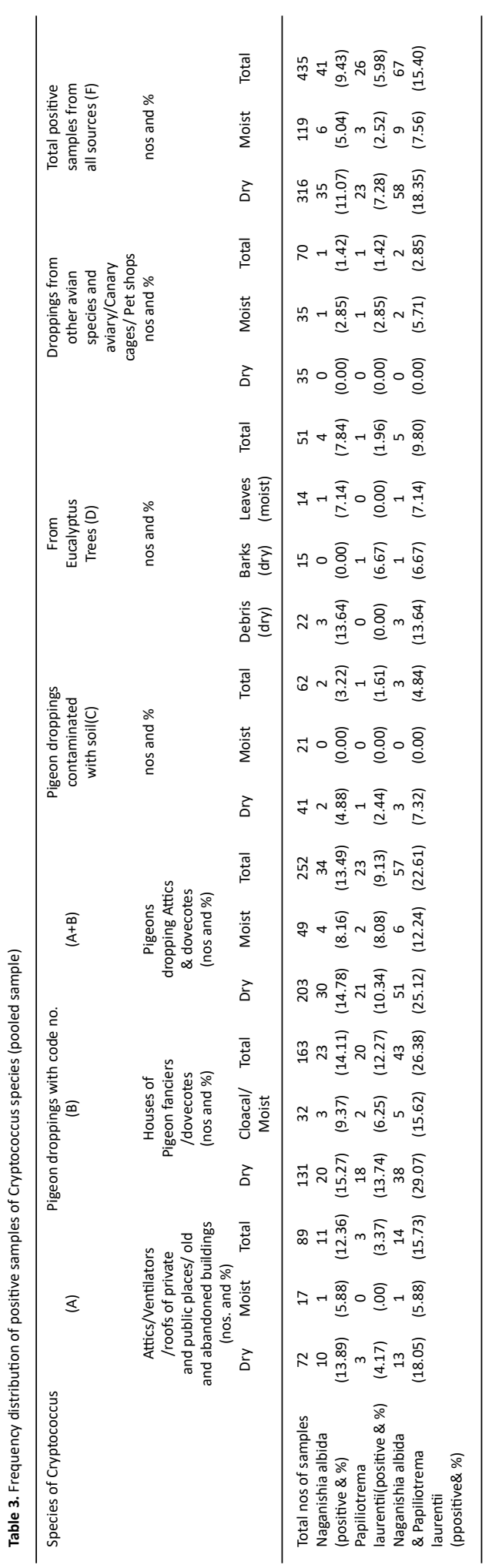

albida is presented in Table 5 whilst morphological features of the species is presented in the Fig 4 (D, E, F) as well as Fig. 6B, and Fig. 8 respectively. Results interpret a significant difference at 0.05 $\%$ level among the different proportions obtained from different sources during the study. On the contrary, the overall prevalence of Papiliotrema laurentii in all dried and moist environmental samples were $5.98 \%$ (26 of 435 ), $7.28 \%$ (23 of 316 ) and $2.52 \%$ (3 of 119) respectively from different sites. Details of the prevalence of the species is presented in Table. 6, Fig. 6(A) and Fig. 9 alongwith the comparable datasheet of reference strain (Cryptococcus laurentii MTCC 2898, Fig. 5). Result correlate a significant difference at 0.05 $\%$ level among the representative proportions obtained from different sources during the study. Details of genetic identity of the isolated strains of Naganishia albida and Papiliotrema laurentii including sequenced data of the rDNA ITS region and their accession numbers obtained from NCBI after submission of the sequences are presented in Table. 7 and Fig. 10 respectively.

Comparisons pertaining to the prevalence of Naganishia albida and Papiliotrema laurentii across the studied geographical locations depict an overall 11 (18.64\%), 16 (17.58\%), 9 (16.98\%), 16 (15.38\%). 10 (13.33\%) and 5 (9.43\%) numbers of positive isolates representing the districts of Bongaigaon, Dhubri, Kokrajhar, Goalpara, Kamrup and Nalbari (Table. 8; Fig. 11). We could not establish any significant difference in the results observed across different districts. Moreover, the prevalence of pathogenic Cryptococcus neoformans could not be observed from any of the tested samples indicating absence of the species in the specimens collected which may be due to unfavorable habitat niche for the species along the lower Brahmaputra valley of Assam resulting in complete absence of the most feared representative form of the family of Cryptococcus.

\section{DISCUSSION}

Cryptococcosis is a major public health concern in India. Epidemiology and pathogenicity of this enigmatic myco-zoonosis in man and animals have been well studied ${ }^{18-21}$. Isolation and identification of Cryptococcus from the environment demonstrates the importance of biodiversity and environmental niche of the pathogenic 


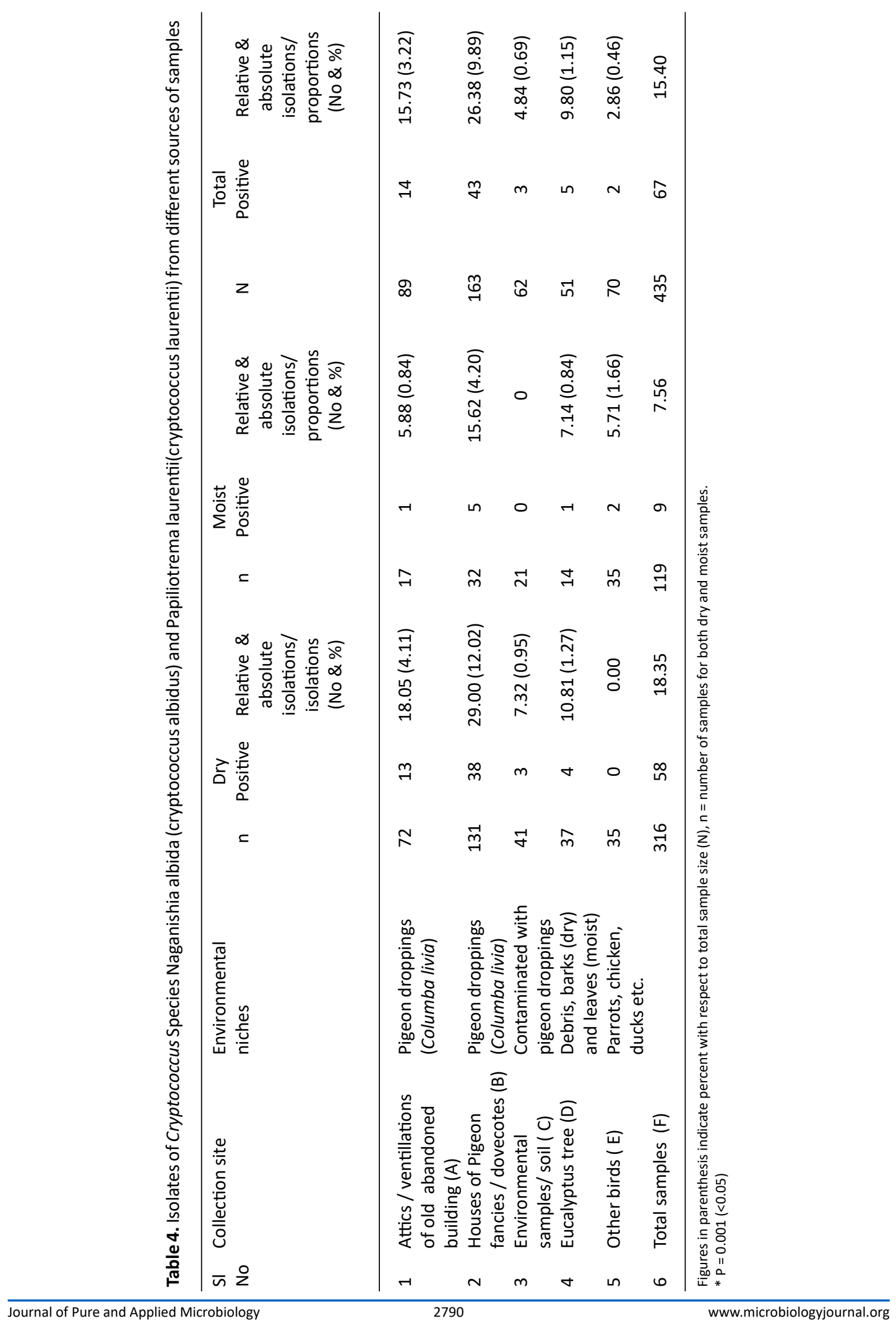




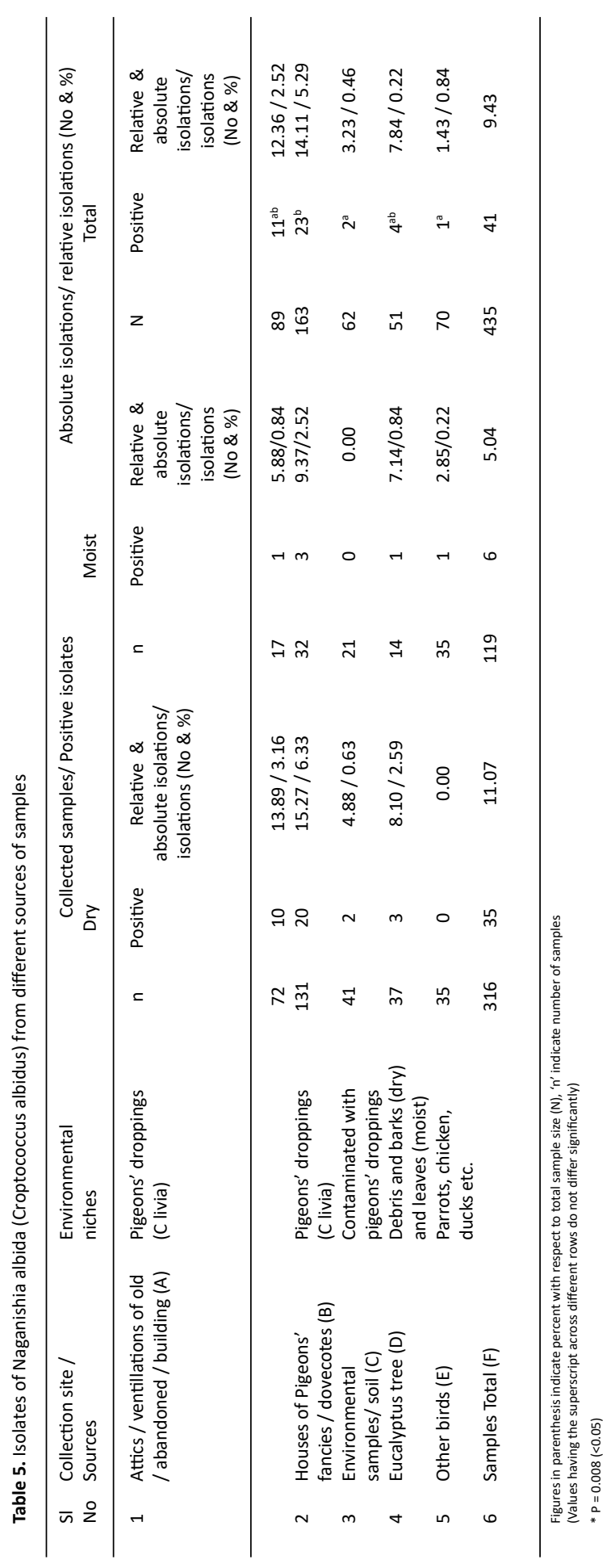


species and strains of this basidiomycota yeast type fungus because such species usually increase the risk of infection in susceptible population exposed to hypersensitive reactions. Besides, vulnerability of infection among the immunocompromised hosts other than the infectious state

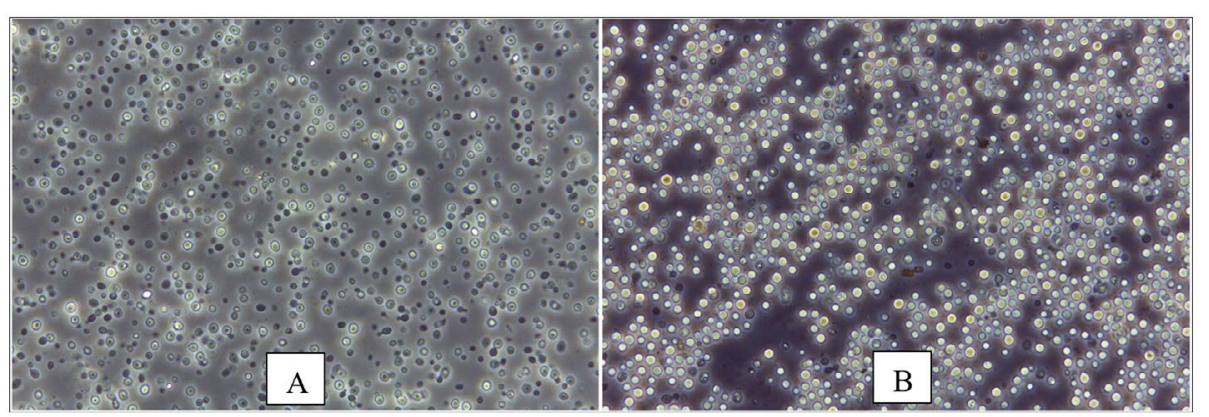

Fig. 6. Phase contrast microscopy of (A) Papiliotrema laurentii (B) Naganishia albida under 40x magnification.

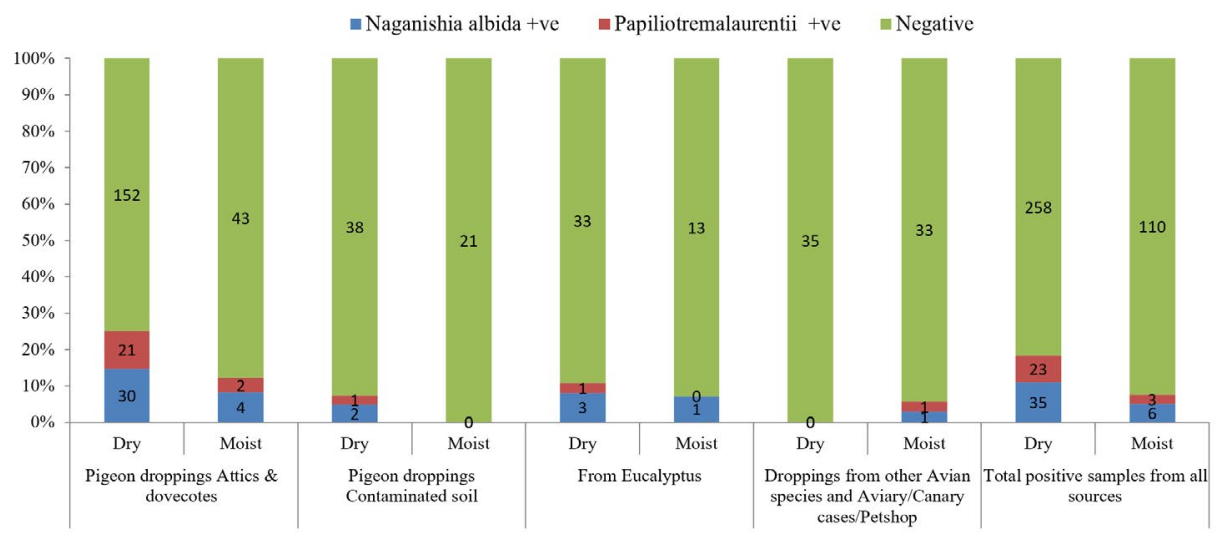

Fig. 7. Distribution of positive samples of Cryptococcus species isolated from samples and specimens

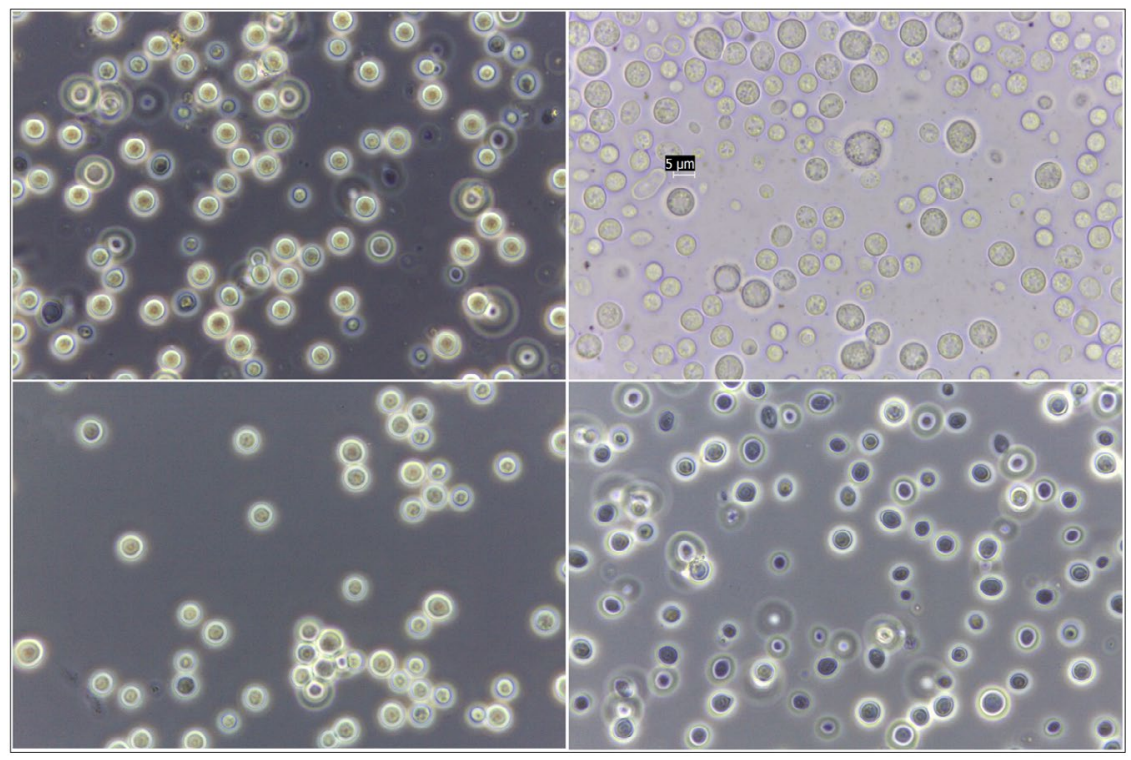

Fig. 8. Phase contrast microscopy of Nagashia albida under 100x magnification

Journal of Pure and Applied Microbiology 


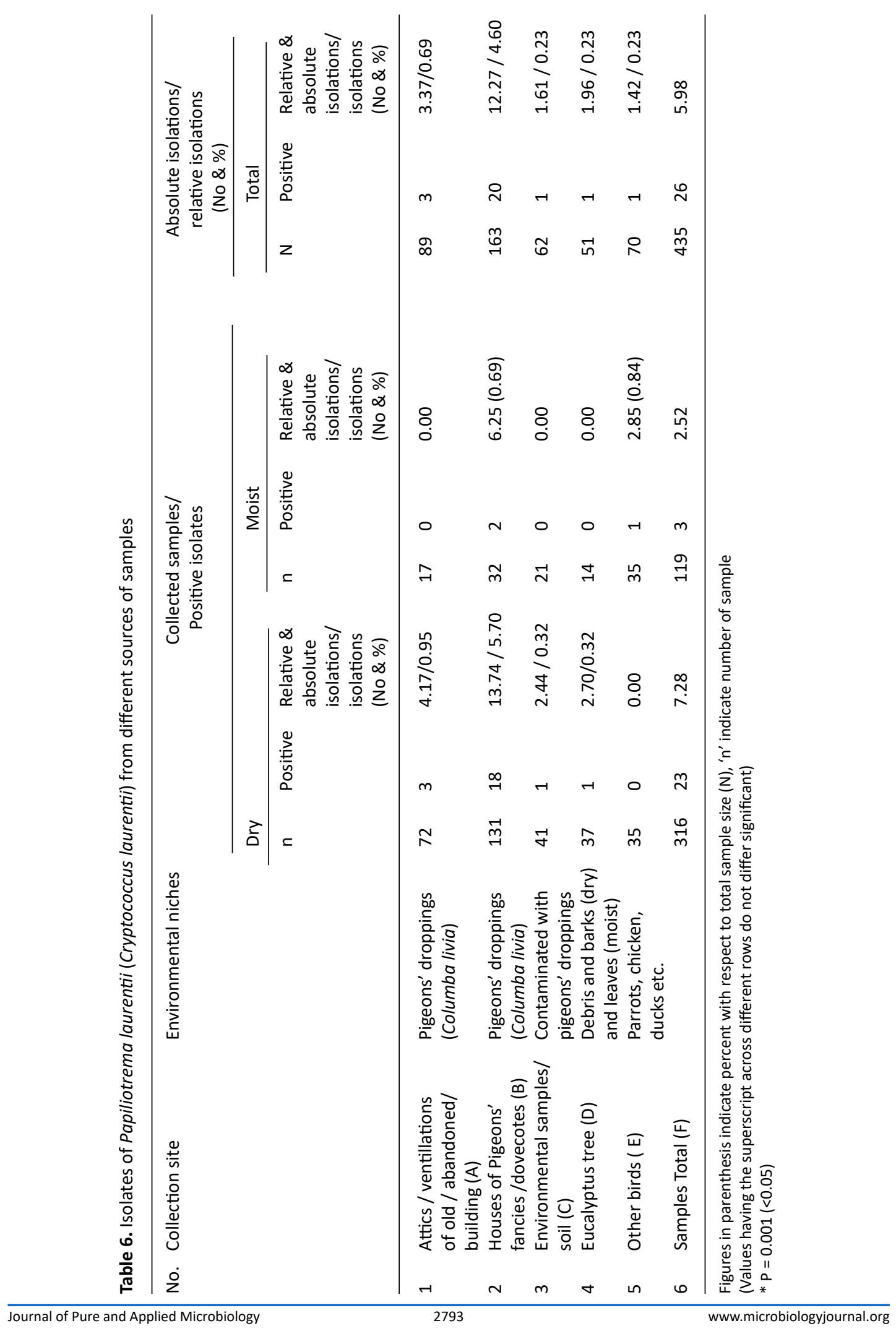


of different biovars of this pathogen, includes both pathogenicity and antifungal resistance which have tremendous implications. Ecological relationship of Cryptococcus was not known until Emmons (1955) ${ }^{10}$ found the fungus in droppings of pigeon and soil colonized by the genera. Later, his observation was substantiated to be true by many researchers $2,3,17,20,24$.

Although many studies have been conducted on the epidemiology and pathogenicity of Cryptococcus neoformans, yet there is scarcity of current information on the prevalence, epidemiology and pathogenicity of the nonneoforman species. The present study defines the incidence of non-neoforman species of the genus Cryptococcus inherent in pigeon droppings, ecological niche of eucalyptus trees and in other microfloral sources of the environment at a specific geographical region along the lower Brahmaputra valley of Assam.

Our observation confirmed the presence of two non-neoforman species of the genus Cryptococcus, viz., Naganishia albida and Papiliotrema laurentii. To our knowledge, this is the first report on the isolation of these two species from pigeon droppings and other environmental sources of the northeastern region of India and this may be considered as an established fact that the environmental sources of the studied areas serve as ideal saprobic reservoirs of these two opportunistic pathogens. Considering the importance of the species at the backdrop

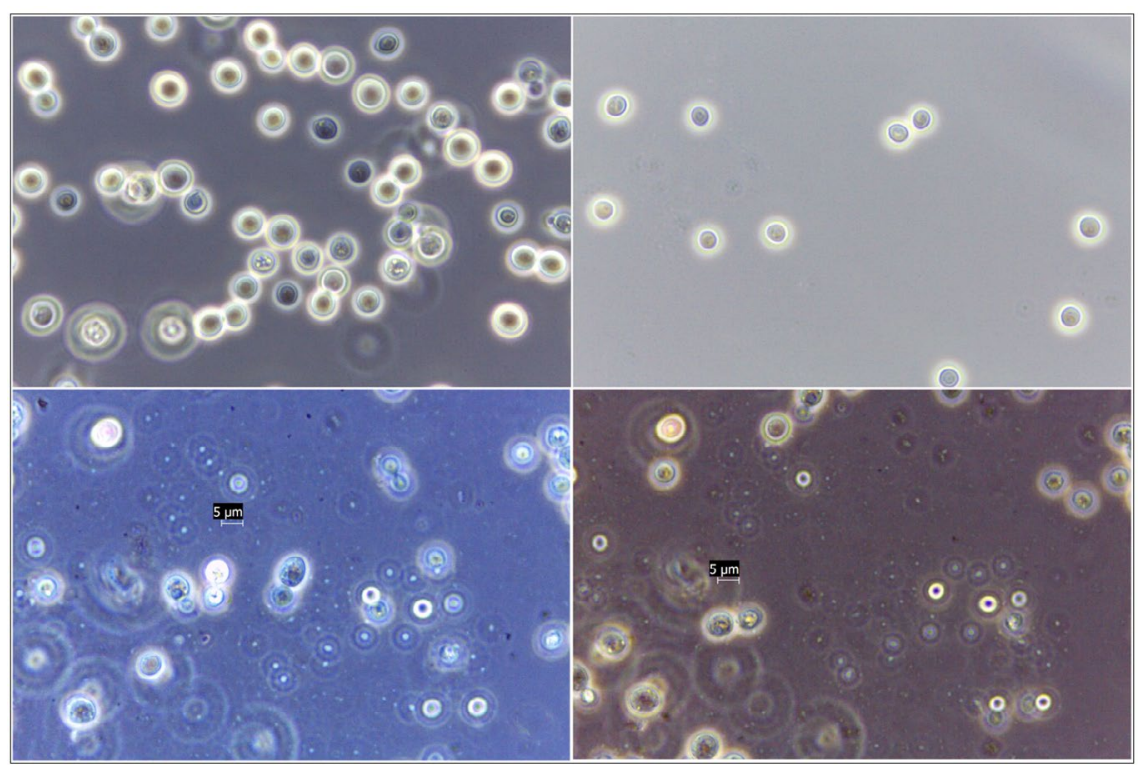

Fig. 9. Phase contrast microscopy of Papiliotrema laurentii under 100x magnification

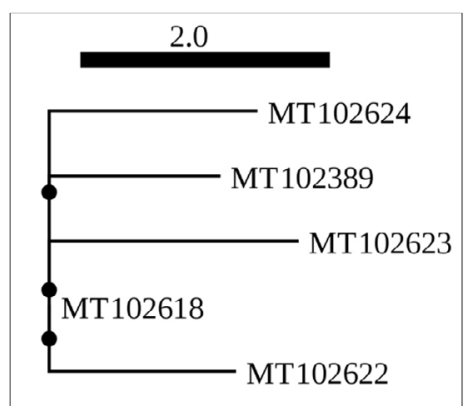

(A)

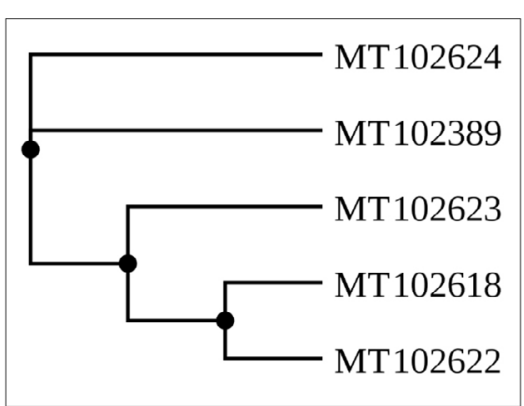

(B)

Fig. 10. (A) Phylogram and (B) Dendrogram prepared by using PhyML version: 3.0 
Islam et al. | J Pure Appl Microbiol | 14(4):2781-2800 | December 2020 | https://doi.org/10.22207/JPAM.14.4.54

Table 7. Sequence data of Cryptococcus species isolated from different sources.

\begin{tabular}{|c|c|c|c|c|c|}
\hline $\begin{array}{l}\text { SI } \\
\text { No }\end{array}$ & $\begin{array}{l}\text { Laboratory } \\
\text { Code }\end{array}$ & Source & Spacies & $\begin{array}{l}\text { Accession } \\
\text { identified } \\
\text { (isolates) }\end{array}$ & $\begin{array}{l}\text { Sequence (in FASTA format) } \\
\text { No }\end{array}$ \\
\hline
\end{tabular}

\begin{tabular}{llll}
\hline GLP 19 & $\begin{array}{l}\text { Pigeon } \\
\text { (Columba livia) } \\
\text { dropping }\end{array}$ & $\begin{array}{l}\text { Naganishia albida } \\
\text { (Cryptococcus } \\
\text { albidus) }\end{array}$ & MT 102389
\end{tabular}

>MT102389.1 Naganishia albida isolate GLP19 small subunit ribosomal RNA gene, partial sequence TTCTGGTGCCCAAGCAGCCGCGGTAATTCCAGCTCCAAT TAGCGTATATTAAAGTTGTTGCAGTTAAAAAGCTCGTA GTTGAACTTCAGGCCCGACGGGGTGGTCTGCCTCACGG TATGTACTATCCGGTTGGGCCTTACCTCCTGGTGAGCC CGTATGTCGTTTACTCGGTGTGCGGGGGAACCAGGA ATTTTACTTTGAAAAAATTAGAGTGTTCAAAGCAGG CATATGCCCGAATACATTAGCATGGAATAATAGAATAG GACGTGCGGTTCTATTTTGTTGGTTTCTAGGATCGCC GTAATGATTAATAGGGACGGTTGGGGGCATTAGTATT CAGTTGCTAGAGGTGAAATTCTTAGATTTACTGAAG ACTAACTACTGCGAAAGCATTTGCCAAGGACGTTTTC ATTAATCAAGAACGAAGGTTAGGGGATCAAAAATGAT TAGATACCGTTGTAGTCCTAACAGTAAACTATGCCGA CTAGGGATCGGGCCATGTTCAACTTTTGACTGGCTCGG CACCTTACGAGAAATCAAAGTCTTTGGGTTCTGGGGG GAGTATGGTCGCAAGGCTGAAACTTAAAGGAATTGA CGGAAGGGCACCACCAGGCGTGGAGCCTGCGGCTT AATTTGACTCAACACGGGGAAACTCACCAGGTCCA GACATAGTAAGGATTGACAGATTGATAGCTCTTTCTTGAT TCTATGGGTGGTGGTGCATGGCCGTTCTTAGTTGGTGGAG TGATTTGTCTGGTTTAATTCCGATAACGAAACGAGACCTTT

\section{$2 \quad$ DHB 39 \\ Evironmental} sample near pigeons' dovecotes (contaminated soil)
Papiliotrema laurentii (Cryptococcus laurentii)
MT 102618 AACCTTGCGTTAGAATTAGGACCCCGGGTTCAGGGGACCT $>$ MT102618.1 Papiliotrema laurentii isolate DHB39 small subunit ribosomal RNA gene, partial sequence TTTCTGGTGCCGCAGCAGCCGCGGTAATTCCAGCTCC AGTAGCGTATATTAAAGTTGCTTGCAGTTAAAAAGCTA GTAGTCGAACTTCGGCCCTGGCTGGACGGTCCGCCTTAC GGTGTGCACTGTCCGGCCGGGTCTTACCCTCTTGGTGAGG CCGCATGCCCTTTACTGGGTGTGCGGTGGAACCAGG AATTTTACCTTGAGAAAATTAGAGTGTTCAAAGCAGGCATTT GCCCGAATACATTAGCACGGAATAATAGAATAGGACGTGCGG TTCTATTTTGTTGGTTTCTAGGATCGCCGTAATGATTAATAG GGACGGTCGGGGGCATTAGTATTCCGTTGCTAGAGGTGAA ATTTTTAGATTTACGGAAGACTAACTTCTGCGAAAGCATTTGC CAAGGACGTTTTCATTGATCAAGAACGAAGGTTAGGGGATCA AAAACGATTAGATACCGTTGTAGTCTTAACAGTAAACTATGCC GACTAGGGATCGGGCCACGTTAATTTCTGACTGGCTCGGCACC TTACGAGAAATCAAAGTCTTTGGGTTCTGGGGGGAGTATGGTC GCAAGGCTGAAACTTAAAGGAATTGACGGAAGGGCACCAC CAGGTGTGGAGCCTGCGGCTTAATTTGACTCAACACGGGG AAACTCACCAGGTCCAGACATAGTAAGGATTGACAGATT GATAGCTCTTTCTTGATTCTATGGGTGGTGGTGCATGGCCG TTCTTAGTTGGTGGAGTGATTTGTCTGGTTAATTCCGA TAACGAACGAGACCTTAACCTGCTAAATAGCCAGGCCGG CTTTGGCTGGTTCCGGTACTTCTTTTACTGGACTGTACGT CTATTATACCCACACTGGATCTGGGACTAACCGCCGGTC GCTAGATCCCCCCTATGAGA

$>$ MT102622.1 Papiliotrema laurentii isolate KRP21 small subunit ribosomal RNA gene, partial sequence TTCCCGCTGCTGCTGCCCGCCCCGCACGCGGCAATCCAGCTC CAGTAGCGTATATTAAAGTTGTTGCAGTTAAAAAGCTCGTA GTCGAACTTCGGGCCTGGCTGGACGGTCCGCCTTACGGTGT GCACTGTCCGGCCGGGTCTTACCTCTTGGTGAGGCCGCATG CCCTTTACTGGGTGTGCGGTGGAACCAGGAATTTTACCTTGA GAAAATTAGAGTGTTCAAAGCAGGCATTTGCCCGAATACATTA GCATGGAATAATAGAATAGGACGTGCGGTTCTATTTTGTTGG TTTCTAGGATCGCCGTAATGATTAATAGGGACGGTCGGGGG CATTAGTATTCCGTTGCTAGAGGTGAAATTCTTAGATTTACGG AAGACTAACTTCTGCGAAAGCATTTGCCAAGGACGTTTTTCAT TGATCAAGAACGAAGGTTAGGGGATCAAAAACGATTAGATA CCGTTGTAGTCTTAACAGTAAACTATGCCGACTAGGGATCGG GCCACGTTAATTTCTGACTGGCTCGGCACCTTACGAGAAATCA AAGTCTTTGGGTTCTGGGGGGAGTATGGTCGCAAGGCTGAA 


\begin{tabular}{|c|c|c|c|c|c|}
\hline & & & & & ACTTAAAGGAATTGACGGAAGGGCACCACCAGGTGTGGAG \\
\hline & & & & & CCTGCGGCTTAATTTGACTCAACACGGGGAAACTCACCAG \\
\hline & & & & & GTCCAGACATAGTAAGGATTGACAGATTGATAGCTCTTTC \\
\hline & & & & & TTGATTCTATGGGTGGTGGTGCATGGCCGTTCTTAGTTGGT \\
\hline & & & & & GGAGTGATTTGTCTGGTTAATTCCGATAACGAACGAGACCTTAA \\
\hline & & & & & CCTGCTAAATAGCCAGGCCGGCTTTGGGCTGGTCGTCGGCTT \\
\hline & & & & & CTTAGAGGGACTGTCGGCGTTTAGCCGACGGAGTTTGAG \\
\hline & & & & & CAATCACAGATATAA \\
\hline \multirow[t]{22}{*}{4} & BNG 4 & $\begin{array}{l}\text { Pigeon } \\
\text { (Columba }\end{array}$ & $\begin{array}{l}\text { Papiliotrema } \\
\text { laurentii }\end{array}$ & MT 102623 & $\begin{array}{l}\text { >MT102623.1 Papiliotrema laurentii isolate BNG4 small } \\
\text { subunit ribosomal RNA gene, partial sequence }\end{array}$ \\
\hline & & livia) & (Cryptococcus & & GCGGTAATTCCAGCTCCAGTAGCGTATATTAAAGTTGTTGCAG \\
\hline & & dropping & laurentii) & & TTAAAAGCTCGTAGTCGAACTTCGGGCCTGGCTGGACGGT \\
\hline & & & & & CCGCCTTACGGTGTGCACTGTCCGGCCGGGTCTTACCTCTT \\
\hline & & & & & GGTGAGGCCGCATGCCCTTTACTGGGTGTGCGGTGGAACC \\
\hline & & & & & AGGAATTTTAACCTTGAGAAAATTAGAGTGTTCAAAGCAGG \\
\hline & & & & & CATTTGCCCGAATACATTAGCATGGAATAATAGAATAGGAC \\
\hline & & & & & GTGCGGTTCTATTTTGTTGGTTTCTAGGATCGCCGTAATGA \\
\hline & & & & & TTAATAGGGACGGTCGGGGGCATTAGTATTCCGTTGCTAGA \\
\hline & & & & & GGTGAAATTCTTAGATTTACGGAAGACTAACTTCTGCGAAA \\
\hline & & & & & GCATTTGCCAAGGACGTTTTCATTGATCAAGAACGAAGGTT \\
\hline & & & & & AGGGGATCAAAAACGATTAGATACCGTTGTAGTCTTAACAG \\
\hline & & & & & TAAACTATGCCGACTAGGGATCGGGCCACGTTAATTTCTGAC \\
\hline & & & & & TGGCTCGGCACCTTACGAGAAATCAAAGTCTTTGGGTTCTGG \\
\hline & & & & & GGGGAGTATGGTCGCAAGGCTGAAACTTAAAGGAATTGAC \\
\hline & & & & & GGAAGGGCACCACCAGGTGTGGAGCCTGCGGCTTAATTTG \\
\hline & & & & & ACTCAACACGGGGAAACTCACCAGGTCCAGACATAGTAAG \\
\hline & & & & & GATTGACAGATTGATAGCTCTTTCTTGATTCTATGGGTGG \\
\hline & & & & & TGGTGCATGGCCGTTCTTAGTTGGTGGAGTGATTTGTCTGGTTA \\
\hline & & & & & ATTCCGATAACGAACGAGACCTTAACCTGCTAAATAGCCA \\
\hline & & & & & GGCCGGCTTTGGCTGGTCGTCGGCTTCTAGAGGGACGT \\
\hline & & & & & CGGCGTTTAGCCGACGGAAGTTTGAGGCAATAACA \\
\hline \multirow[t]{22}{*}{5} & KJR 27 & Pigeon & Naganishia & MT 102624 & >MT102624.1 Naganishia albida isolate KJR27 small \\
\hline & & (Columba livia) & albida & & subunit ribosomal RNA gene, partial sequence \\
\hline & & dropping & (Cryptococcus & & AGCTCCAATAGCGTATATTAAAGTTGTTGCAGTTAAAAAA \\
\hline & & & albidus) & & GCTCGTAGTTGAACTTCAGGCCCGACGGGGTGGTCTGCCT \\
\hline & & & & & CACGGTATGTACTATCCGGTTGGGCCTTACCTCCTGGTGAG \\
\hline & & & & & CCCGTATGTCGTTTACTCGGTGTGCGGGGGAACCAGGA \\
\hline & & & & & ATTTTACTTTGAAAAAATTAGAGTGTTCAAAGCAGGCATAT \\
\hline & & & & & GCCCGAATACATTAGCATGGAATAATAGAATAGGACGTGCG \\
\hline & & & & & GTTCTATTTTGTTGGTTTCTAGGATCGCCGTAATGATTAATA \\
\hline & & & & & GGGACGGTTGGGGGCATTAGTATTCAGTTGCTAGAGGTGA \\
\hline & & & & & AATTCTTAGATTTACTGAAGACTAACTACTGCGAAAGCATT \\
\hline & & & & & TGCCAAGGACGTTTTCATTAATCAAGAACGAAGGTTAGGG \\
\hline & & & & & GATCAAAAATGATTAGATACCGTTGTAGTCCTAACAGTAAA \\
\hline & & & & & CTATGCCGACTAGGGATCGGGCCATGTTCAACTTTTGACTG \\
\hline & & & & & GCTCGGCACCTTACAAAAAATCAAATTCTTTGGGTTCTGG \\
\hline & & & & & GGGAAGTATGGTCGCAAGGCTGAACCTTAAAAGAATTGAC \\
\hline & & & & & GGAGGGGCACCACCAGGCTTGAAGCCGGCGGCTTATTTTA \\
\hline & & & & & ACTCATCACGGGGAAACTCACCAGGTCCGACACATACTAG \\
\hline & & & & & GATTGACACATTGATATTTCTTTCTTGATTCTCTGGGTGGCGGTG \\
\hline & & & & & CATGCCCGTTCTAAGTTGGTGGAGTGATTTGTCTGGTTAATG \\
\hline & & & & & CCCAATCACGAACGTACATCTTAACCTGCTAACTACACCGA \\
\hline & & & & & TCGGGCTTTGAGCTGCACCCTCTATTCTTTA \\
\hline
\end{tabular}

of health status of man and animals, these observations may be considered important as the prevalence of cryptococcosis is currently at a rising trend and an increase in the cases of fungemia both in human and animals have been on the rise in the past few years. However, in the current investigation, not a single isolate of Cryptococcus neoformans was recovered despite our incessant attempts to culture the isolate in appropriate Bird Seed Agar and Sabouraud Dextrose Agar media as per international standards.
The overall prevalence of Cryptococcus spp. was $15.40 \%$ (67) retrieved from all six geographical regions studied. Of the 67 positive isolates, $9.43 \%(41)$ and $5.98 \%$ (26) were identified to represent Naganishia albida and Papiliotrema laurentii respectively. The results are comparable with other findings reported by Jang et al $(2011)^{26}$ who stated the occurrence of $14.30 \%$ Cryptococcus albidus and $7.9 \%$ Cryptococcus laurentii in pigeon droppings from China. In another study, Kamari et.al, $(2017)^{16}$ reported 33 
of $186(17.7 \%)$ positive cultures and 11 out of 88 (12.5\%) confirmed cultures of Cryptococcus spp. retrieved from pigeon nests and eucalyptus tree specimens from llam province in Iran. The authors also documented the prevalence of Cryptococcus albidus (17.2\%), C. albidus var. kuetzingii (3.4\%), C. adilensis (3.4\%), C. uzbekistanensis (3.4\%) and C. neoformans var grubii (3\%) from pigeon nests while they concluded the presence of only $C$. adilensis (25\%) in specimens from eucalyptus trees. .

The finding of 23 (14.11\%) Cryptococcus in pigeon droppings from the dovecotes / houses of pigeon fanciers was comparable with the study of Kamari et. al, (2017) $)^{16}$. Our findings revealed $21(10.34 \%)$ positive isolates of Papiliotrema laurentii in the droppings of pigeon in dovecotes / fanciers whereby 4 out of 51 (7.84\%) and 1 (1.96\%) represented Naganishia albida and Papiliotrema laurentii respectively. Similarly, $20.60 \%$ positive isolation rate of Cryptococcus in 6 out of 186 pigeon droppings in attics was reported earlier by Kamariet.al, (2017). In this study, 38 out of $89(15.73 \%)$ isolates from attics were positive for Cryptococcus spp. The slight variation of the findings might be due to differences of climate, humidity, temperature and other biotic or abiotic factors characteristic of both the countries that are geographically apart and separated by a huge landmass.

In the current study, $13.49 \%$ (34) and 9.13\% (23) of Naganishia albida and Papiliotrema laurentii could be recovered from pigeon droppings from attic ventilations of old buildings, dovecotes houses of pigeon fanciers and from cloacal swabs. Earlier, Rosario et. al. (2009) ${ }^{24}$ reported a lower isolation rate of 23 (6.9\%) Cryptococcus albidus and $2(0.6 \%)$ Cryptococcus laurentii from droppings of pigeons in Spain which may be due to differences in environmental and climatic conditions as mentioned above. Studies have also been reported from Nigeria whereby Pal (2015) ${ }^{47}$, had isolated 16 representatives of Cryptococcus neoformans from environmental samples of pigeon droppings with a prevalence of $12.5 \%$. In the present study, the prevalence of Naganishia albida (13.49\%) and Papiliotrema laurentii $(9.13 \%)$ in pigeon droppings and in eucalyptus tree specimens were comparable to previous reported findings, despite the absence of Cryptococcus neoformans.

Although Cryptococcus neoformans is regarded as the most commonly occuring species of the Cryptococcus family, its absence in probable specimens collected from this part of the country is quite intriguing and it reflects the status of overall persistence of the species under humid conditions exposed to abrupt change in temperatures, a feature commonly observed in the studied locations. . The effect of seasonal variations on the persistence of non-neoforman Cryptococcus isolates cannot be nullified and it suggest the need of proper screening through a robust molecular approach concomitant to the behavior and food sources of the birds questioned, not to mention

Table 8. Geographical co-ordinates and environmental niches wise frequency distribution of positive isolates of Cryptococcus species

\begin{tabular}{|c|c|c|c|c|c|c|c|}
\hline $\begin{array}{l}\text { SI } \\
\text { No. }\end{array}$ & $\begin{array}{l}\text { Geographical } \\
\text { region }\end{array}$ & $\begin{array}{l}\text { Total } \\
\text { Sample }\end{array}$ & $\begin{array}{l}\text { Naganishia } \\
\text { albida } \\
\text { positive } \\
\text { (nos.) }\end{array}$ & $\begin{array}{l}\text { Papiliotrema } \\
\text { laurentii } \\
\text { positive } \\
\text { (nos.) }\end{array}$ & $\begin{array}{l}\text { Total } \\
\text { positive } \\
\text { sample } \\
\text { (nos.) }\end{array}$ & $\begin{array}{l}\text { Relative \% } \\
\text { in the } \\
\text { region }\end{array}$ & $\begin{array}{l}\text { Absolute \% } \\
\text { in the } \\
\text { region }\end{array}$ \\
\hline 1 & BONGAIGAON & 59 (BNG 1-59) & 6 & 5 & 11 & 18.64 & 2.53 \\
\hline 2 & KOKRAJHAR & 53 (KJR 1-53) & 6 & 3 & 9 & 16.98 & 2.06 \\
\hline 3 & DHUBRI & 91 (DHB 1-91) & 10 & 6 & 16 & 17.58 & 3.68 \\
\hline 4 & KAMRUP & 75 (KRP 1-75) & 6 & 4 & 10 & 13.34 & 2.99 \\
\hline 5 & GOALPARA & 104 (GLP 1-104) & 10 & 6 & 16 & 15.38 & 3.68 \\
\hline \multirow[t]{3}{*}{6} & NALBARI & 53 (NLB 1-53) & 3 & 2 & 5 & 9.43 & 1.15 \\
\hline & & & & & \multicolumn{3}{|c|}{$P=0.762(>0.05): N S$} \\
\hline & & TOTAL = 435 & Total $=41$ & Total $=26$ & 67 & $15.04 \%$ & \\
\hline
\end{tabular}

NS: indicate non-significant 
the presumable role of temperature and other edaphic factors that needs further appreciation. Isolation of several species and varieties of the genus Cryptococcus from different sources, soils, atmospheric airs, dust and even from other avian species i.e chicken, ducks, parrots and munia had been previously reported by many authors. In a study conducted in Brazil, Leite et. al, (2012) ${ }^{48}$ found the occurrence of 18 (21.4\%) Cryptococcus species from 84 specimens of dust collected from public libraries. The isolation rate of Cryptococcus albidus $2(4.6 \%)$ in their study was found to match our findings from dry soil 2 (4.88\%). Furthermore, the percentage of isolation of Cryptococcus species in this study was $18.33 \%(58)$ as compared to $7.56 \%$ (9) from a total of 435 collected specimens in dry and moist conditions. The observations were in congruence with the number of isolates from pigeon droppings whereby $25.12 \%$ (51) and $12.24 \%$ (6) Cryptococcus isolates could be recovered from dry and moist specimens.

While studying the frequency of isolation in context of regional characteristics, no specific trend in terms of frequency was observed as the number of isolates varied from $9.43 \%$ (in Nalbari region) to $18.64 \%$ (Bongaigaon region), despite close proximity of the study areas that share the same environmental conditions. It may be recalled that factors like humidity, rainfall, temperature and $\mathrm{pH}$ of a definite geographical location do affect population parameters of domesticated birds like pigeons which need further evaluation. Ecological relevance of the non-neoformans species of
Cryptococcus (4.84\% in soil, $9.80 \%$ in eucalyptus tree samples) was the most important findings of this study with profound influence on replication and transmission of the pathogenic species from the ideal sources to the susceptible population at risk. Such situation is very imminent and highly dangerous for crowded and highly populated areas like Nalbari and Kamrup as the route of entry of such microorganisms is either through inhalation or direct skin contact.

From this investigation, it may be concluded that Naganishia albida and Papiliotrema laurentii are the most prevalent Cryptococcus species in the lower Assam belt compared to the more pathogenic Cryptococcus neoformans indicating the need of extensive epidemiological studies for establishing proper identify of the different strains that perpetuate, thrive and adapt to changing environmental conditions which are bound to challenge public health and pose as potential hazards in the near future. It may be stated that to ensure safety of the people, mass awareness programmes on the prevalence of the fungus need to be arranged whilst proper prevention, control and management of such disease causing pathogens be prioritized to prevent future outbreaks so as to minimize the risk of transmission of opportunistic Cryptococcus infections.

\section{ACKNOWLEDGMENTS}

The authors duly acknowledge the support and contribution provided by Department

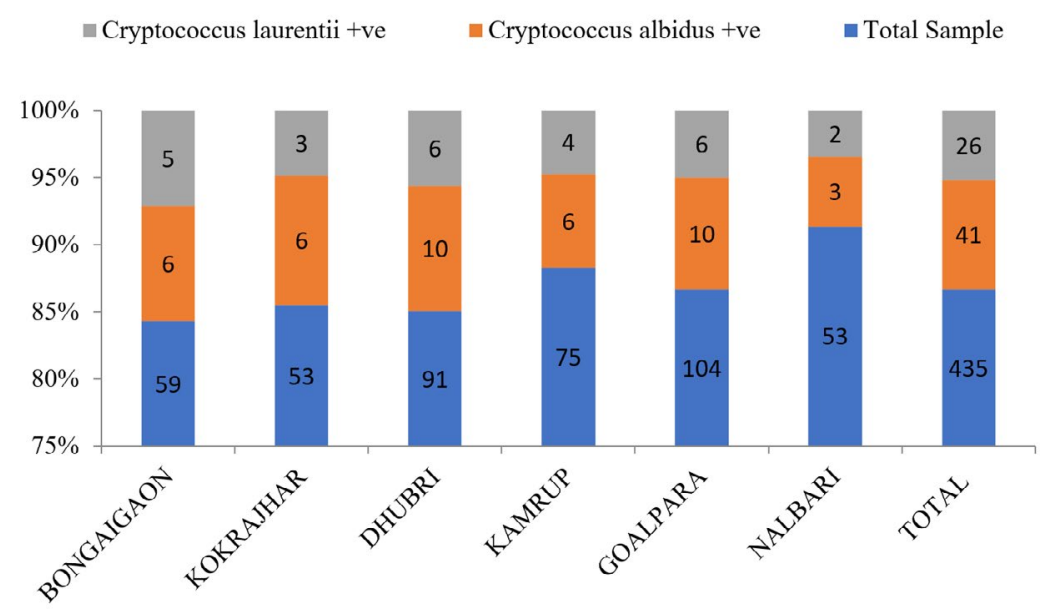

Fig. 11. District wise distribution of positive isolates of Cryptococcus species 
of Biotechnology, Gauhati University, Guwahati, Assam to carry out the research activity. The authors also duly acknowledge the support and cooperation received from state department of Animal husbandry and Veterinary, Govt of Assam, Xcelris Labs Limited and other personnel involved during the data collection / processing to carry out task effectively.

\section{CONFLICT OF INTEREST}

The authors declare that there is no conflict of interest.

\section{AUTHORS' CONTRIBUTIONS}

All authors listed have made a substantial, direct and intellectual contribution to the work, and approved it for publication.

\section{FUNDING}

None.

\section{DATA AVAILABILITY}

As per the research design, data were collected from different primary sources / generated are available with the researcher and NCBI database (Accession No: MT 102389, MT 102618, MT 102622, MT 102623, MT 102624)

\section{ETHICS STATEMENT}

Not applicable. However, during the sample collection from pigeons, proper handling was done as per the standard veterinary practices.

\section{REFERENCES}

1. Lewis $\mathrm{JL}$, Rabinovich $\mathrm{S}$. The wide spectrum of cryptococcal infections. Am J Med. 1972;53(3):315322. doi: 10.1016/0002-9343(72)90174-X.

2. Littman ML. Cryptococcosis (Torulosis). Current concepts and therapy. Am J Med. 1959;27(6):976-998. doi: 10.1016/0002-9343(59)90181-0.

3. Pal M, Dave P. Cryptococcosis: An Emerging Airborne Mycosis of Global Concern. Air \& Water Borne Diseases. 2016;5(1):1000127. doi: 10.4172/2167 7719.1000127.

4. Kwon-Chung KJ, Boekhout T, Wickes BL, Fell JW. Systematics of the Genus Cryptococcus and Its Type Species C. neoformans. Cryptococcus. 2014.

5. Cheng MF, Chiou CC, Liu YC, Wang HZ, Hsieh KS. Cryptococcus laurentii fungemia in a premature neonate. J Clin Microbiol. 2001;39(4):1608-1611. doi: 10.1128/JCM.39.4.1608-1611.2001

6. Darze C, Lucena R, Gomes I, Melo, A. Clinical and laboratory characteristics of 104 Cryptococcus meningoencephalitis cases. Revista Da Sociedade Brasileira de Medicina Tropical. 2000.

7. Rosario I, Acosta B, Colom F. La paloma y otras aves como reservorio de Cryptococcus spp Pigeons and other birds as a reservoir for Cryptococcus spp. Revista Iberoamericana de Micologia. 2008;25(1):S13-S18. doi: 10.1016/S1130-1406(08)70020-2

8. Emmons CW. Isolation of Cryptococcus neoformans from soil. J Bacteriol. 1951;62(6):685-690. doi: 10.1128/JB.62.6.685-690.1951

9. Emmons CW. Prevalence of Cryptococcus neoformans in pigeon habitats. Public Health Rep. 1960:75:362364. doi: $10.2307 / 4590800$

10. Emmons CW. Saprophytic sources of Cryptococcus neoformans associated with the pigeon (Columba livia). Am J Epidemiol. 1955;62(3):227-232. doi: 10.1093/oxfordjournals.aje.a119775

11. Ruiz A, Velez D, Fromtling RA. Isolation of saprophytic Cryptococcus neoformans from Puerto Rico: Distribution and variety. Mycopathologia. 1989;106(3):167-170. doi: 10.1007/BF00443058

12. Fromtling RA, Abruzzo GK, Ruiz A. Virulence and antifungal susceptibility of environmental and clinical isolates of Cryptococcus neoformans from Puerto Rico. Mycopathologia. 1989;106(3):163-166. doi: 10.1007/ BF00443057

13. Lazera MS, Cavalcanti MAS, Londero AT, Trilles L, Nishikawa MM, Wanke B. Possible primary ecological niche of Cryptococcus neoformans. Med Mycol. 2000;38(5):379-383. doi: 10.1080/mmy.38.5.379.383

14. Filiu WFdeO, Wankelll B, Aguena SM, et al. Cativeiro de aves como fonte de Cryptococcus neoformans na cidade de Campo Grande, Mato Grosso do Sul, Brasil. Revista da Sociedade Brasileira de Medicina Tropical. 2002;35(6). doi: 10.1590/S0037-86822002000600008

15. Kumar CPG, Prabu D, Mitani H, Mikami Y, Menon T. Environmental isolation of Cryptococcus neoformans and Cryptococcus gattii from living trees in Guindy National Park, Chennai, South India: Letter to the editor. Mycoses. 2010;53(3):262-264. doi: 10.1111/j.1439-0507.2009.01699.x

16. Kamari A, Sepahvand A, Mohammadi R. Isolation and molecular characterization of Cryptococcus species isolated from pigeon nests and Eucalyptus trees. J Mycol Med. 2017;3(2):20-25. doi: 10.29252/ $\mathrm{cmm}$.3.2.20

17. Pal M. Cryptococcus gattii: an emerging global mycotic pathogen of humans and animals. J Mycopathol Res. 2014.

18. Pal M, Randhawa HS. Caprine mastitis due to cryptococcus neoformans. Med Mycol. 1976;14(3):261263. doi: $10.1080 / 00362177685190391$

19. Pal M, Mehrotra BS. Ciyptococcal Mastitis in Daily Animals/Cryptococcen-Mastitis bei Milchvieh. Mycoses. 1983;26(12):615-616. doi: 10.1111/j.14390507.1983.tb03171.x

20. Pal M. First report of isolation of Cryptococcus neoformans var. neoformans from avian excreta in Kathmandu, Nepal. Revista Iberoamericana de Micologia. 1997.

21. Mitchell TG, Perfect JR. Cryptococcosis in the Era of 
AIDS-100 Years after the Discovery of Cryptococcus neoformans. Clin Microbiol Rev. 1995;8(4):515-548. doi: 10.1128/CMR.8.4.515-548.1995

22. Casadevall A, Mukherjee J, Scharff MD. Monoclonal antibody based ELISAs for cryptococcal polysaccharide. J Immunol Methods. 1992;154(1):27-35. doi: 10.1016/0022-1759(92)90209-C

23. Chander J, Sapra RK, Talwar P. Incidence of cryptococcosis in and around Chandigarh, India, during the period 1982-91. Mycoses. 1994;37:23-26. doi: 10.1111/j.1439-0507.1994.tb00280.x

24. Rosario I, de Mendoza MH, Deniz S, Soro G, Alamo I, Acosta B. Isolation of Cryptococcus species including $C$. neoformans from cloaca of pigeons. Mycoses. 2005:48(6):421-424. doi: 10.1111/j.14390507.2005.01153.x

25. Bernardo FM, Martins HM, Martins L. Fontes urbanas de Cryptococcus spp - Lisboa Urban Sources of Cryptococcus spp - Lisbon ( Portugal ). Revista Portuguesa de Ciencias Veterinarias. 2001.

26. Jang YH, Lee SJ, Lee JH, Chae HS, Kim SH, Choe NH. Prevalence of yeast-like fungi and evaluation of several virulence factors from feral pigeons in Seoul, Korea. Lett Appl Microbiol. 2011;52(4):367- 371. doi: 10.1111/j.1472-765X.2011.03009.x

27. Liu Y, Ma S, Wang X, Xu W, Tang J. Cryptococcus albidus encephalitis in newly diagnosed HIV-patient and literature review. Med Mycol Case Rep. 2014;3:8-10. doi: 10.1016/j.mmcr.2013.11.002

28. Huang YH, Lin IH, Chang TC, Tseng SH. Early diagnosis and successful treatment of cryptococcus albidus keratitis. Medicine (United States). 2015;94(19):e885. doi: 10.1097/MD.0000000000000885

29. Averbuch $D$, Boekhout $T$, Falk R, et al. Fungemia in a cancer patient caused by fluconazole-resistant Cryptococcus laurentii. Med Mycol. 2002;40(5):479484. doi: $10.1080 /$ mmy.40.5.479.484

30. Shankar EM, et al. Pneumonia and pleural effusion due to Cryptococcus laurentii in a clinically proven case of AIDS. Can Respir J. 2006;13(5):275-278. doi: 10.1155/2006/160451

31. von Geldern $G$, Nath A. Central nervous system infections. in Evidence-Based Neurology: Management of Neurological Disorders: Second Edition. 2015. doi: 10.1002/9781119067344.ch15

32. Criseo G, Bolignano MS, de Leo F, Staib F. Evidence of canary droppings as an important reservoir of Cryptococcus neoformans. Zentralblatt fur Bakteriologie. 1995;282(3):244-254. doi: 10.1016/ S0934-8840(11)80124-6

33. Liu Z, Ma L, Zhong Y, Wang X, Xie S. Isolation, identification and significance of Cryptococcus neoformans and Candida albicans from faecal specimen of pigeon. Advances in Intelligent and Soft Computing. 2012;134:507-512. doi: 10.1007/978-3642-27537-1_63

34. Grossgebauer K. Detection of cryptococcus neoformans capsule using fluorescent, mucopolysaccharidebinding dyes. Klin Wochenschr. 1980;58(18):943-945. doi: 10.1007/BF01477052

35. Chae HS, Park GN, Kim SH, et al. Rapid direct identification of Cryptococcus neoformans from pigeon droppings by nested PCR using CNLAC1 gene. Poultry Science. 2012;91(8):1983-1989. doi: 10.3382/ps.201202307

36. Filion T, Kidd S, Aguirre K. Isolation of Cryptococcus laurentii from Canada Goose guano in rural upstate New York. Mycopathologia. 2006;162(5):363-368. doi: 10.1007/s11046-006-0066-1

37. Mseddi F, Jarboui M, Sellami A, Sellami H, Ayadi A. $A$ rapid and easy method for the DNA extraction from Cryptococcus neoformans. Biol Proced Online. 2011;13(1):5. doi: 10.1186/1480-9222-13-5

38. Tendulkar SR, Gupta A, Chattoo BB. A simple protocol for isolation of fungal DNA. Biotechnol Lett. 2003;25(22):1941-1944. doi: 10.1023/B:BILE.0000003990.27624.04

39. Motkova P, Vytrasova J. Comparison of methods for isolating fungal DNA. Czech Journal of Food Sciences. 2011;29:s76-s85. doi: 10.17221/266/2011-CJFS

40. Leal AL, Faganello J, Bassanesi MC, Vainstein MH. Cryptococcus species identification by multiplex PCR. Med Mycol. 2008;46(4):377-383. doi: 10.1080/13693780701824429

41. Lau A, Chen S, Sorrell T, et al. Development and clinical application of a panfungal PCR assay to detect and identify Fungal DNA in tissue specimens. J Clin Microbiol. 2007;45(2):380-385. doi: 10.1128/ JCM.01862-06

42. Mitchell TG, Freedman EZ, White TJ, Taylor JW. Unique oligonucleotide primers in PCR for identification of Cryptococcus neoformans. J Clin Microbiol. 1994;32(1):253-255. doi: 10.1128/JCM.32.1.253255.1994

43. Sidrim JJC, Costa ANF, Cordeiro RA, et al. Molecular methods for the diagnosis and characterization of cryptococcus: A review. Can J Microbiol. 2010;56(6):445-458. doi: 10.1139/W10-030

44. XLSTAT. Statistical Power for Repeated Measures Anova. Adinsoft. 2015.

45. Barbosa AM, Santos BFdeO, Carvalho EdEO, et al. Biological activity of Cryptococcus neoformans and Cryptococcus gattii from clinical and environmental isolates. Jornal Brasileiro de Patologia e Medicina Laboratorial. 2013;49(3). doi: 10.1590/S167624442013000300002

46. Adinsoft S. XLSTAT-software, version 10. Addinsoft, Paris, France. 2010.

47. Pal M. First Record of Isolation of Cryptococcus neoformans from Pigeon Droppings in Djibouti. Molecular Microbiology Research. 2015;5(3):1-3. doi: 10.5376/mmr.2015.05.0003

48. Leite DP, Amadio JVRS, Martins ER, et al. Cryptococcus spp isolated from dust microhabitat in Brazilian libraries. Journal of Occupational Medicine and Toxicology. 2012;7(1)11. doi: 10.1186/1745-6673-7-11 